\title{
APPROXIMATION OF STRICTLY SINGULAR AND STRICTLY COSINGULAR OPERATORS USING NONSTANDARD ANALYSIS
}

\author{
BY \\ J. W. BRACE( $\left.{ }^{1}\right)$ AND R. ROYCE KNEECE
}

\begin{abstract}
The strictly singular operators and the strictly cosingular operators are characterized by the manner in which they can be approximated by continuous linear operators of finite-dimensional range. We make use of linear convergence structures to obtain each class as limit points of the operators with finite-dimensional range. The construction of a nonstandard model makes it possible to replace convergence structures by topologies. Our nonstandard models are called nonstandard locally convex spaces.
\end{abstract}

A recent paper [4] showed that classes of "compact-like" operators, including compact, weakly compact, and completely continuous operators, can be characterized by "approximation topologies", i.e. topologies on the space of operators for which the given class is precisely the closure (or completion) of the continuous finite-dimensional range operators. In this paper, techniques providing a similar characterization for strictly singular and strictly cosingular operators are given. It is necessary, however, to generalize the concept of an approximation topology to include convergence structures ([5], [7]), after which it is possible to obtain these two classes of operators as limit points of the finite-dimensional range operators. Furthermore, these convergence structures have a characterization in terms of a nonstandard model ([14], [15]) of the operator space, and in this setting it is possible to obtain topological convergence.

The paper is presented in three sections. $\$ 1$ summarizes characterizations of strictly singular and strictly cosingular operators due to Kato [11], Goldberg [8], Pełczyński [13], and Vladimirskiǐ [18], and some additional results are obtained utilizing nuclear operators. $\$ 2$ contains the characterizations of the strictly singular and strictly cosingular operators as the limit points of the set of continuous finitedimensional range operators under certain linear convergence structures. To obtain

Presented to the Society, January 23, 1970 under the title Approximation of strictly singular operators; received by the editors March 5, 1971 and, in revised form, November 22, 1971.

AMS 1970 subject classifications. Primary 46A99, 47A65, 47B99, 54J05; Secondary 02H25, 54A05, 54A20.

Key words and phrases. Approximation of linear operators, strictly singular operators, strictly cosingular operators, nonstandard analysis, convergence structure, nuclear operators, compact operator.

(1) The first author was partially supported by the National Science Foundation, GP 19 E62. Copyright (C) 1972, American Mathematical Society 
these results, heavy use is made of machinery in [4]. The nonstandard model for the situation is presented in $\S 3$, and the linear convergence structures emerge as nonstandard locally convex topologies. Although some familiarity with nonstandard notions ([14], [15]) would be useful to the reader, this section is largely selfcontained. The strictly singular (or strictly cosingular) operators are shown to be all standard points in the closure of the nonstandard finite-dimensional range operators. Readers not familiar with convergence structures will need to note only Definition 2.4 before reading $\$ 3$. Theorem 3.10 states Theorem 3.6(iii) without reference to convergence structures.

If $N$ is a subspace of a space $X, i_{N}$ will denote the injection mapping of $N$ into $X$, and $\phi_{N}$ will denote the quotient mapping of $X$ onto $X / N$. If $T$ is a bounded linear operator of $X$ into $Y$, then $T^{\prime}$ will denote the adjoint mapping of $Y^{\prime}$ into $X^{\prime}$. For a set $M$ in $X, M^{\perp}$ denotes the set of members of $X^{\prime}$ which are identically zero on $M$. For $M$ a subset of $X^{\prime}, M_{\perp}$ denotes the null space of $M$ in $X$. If $M$ is a closed subspace of $X$, then the adjoint map $\phi_{M}^{\prime}:(X / M)^{\prime} \rightarrow X^{\prime}$ is an isomorphism (by which we mean a linear homeomorphism not necessarily onto) with range precisely $M^{\perp}$. If we define the map $\psi_{M}$ so that the diagram

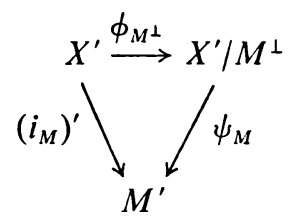

commutes, then $\psi_{M}$ is an isomorphism. The dimension of $M$ is denoted by $\operatorname{dim}(M)$, and the dimension of $X / M$ is denoted by codim $(M)$. If $M$ is a closed subspace of $X$ with $\operatorname{dim}(M)=\infty(\operatorname{codim}(M)=\infty)$ then $M^{\perp}$ is a $\sigma\left(X^{\prime}, X\right)$-closed subspace of $X^{\prime}$ with $\operatorname{codim}\left(M^{\perp}\right)=\infty\left(\operatorname{dim}\left(M^{\perp}\right)=\infty\right)$. If $M$ is a $\sigma\left(X^{\prime}, X\right)$-closed subspace of $X^{\prime}$ with $\operatorname{dim}(M)=\infty(\operatorname{codim}(M)=\infty)$ then $M_{\perp}$ is a closed subspace of $X$ with $\operatorname{codim}\left(M_{\perp}\right)=\infty\left(\operatorname{dim}\left(M_{\perp}\right)=\infty\right)$.

\section{The strictly singular and strictly cosingular operators.}

1.1. TheOREM. Let $T$ be a bounded linear operator mapping the Banach space $X$ into the Banach space $Y$. The following statements are equivalent:

1. (a) $T$ is strictly singular (Kato [11]), i.e. there exists no subspace $N$ of $X$ with $\operatorname{dim}(N)=\infty$ such that $T \circ i_{N}$ is an isomorphism.

(b) Tis strictly singular (Petczyński [13]), i.e. for no infinite-dimensional Banach space $Z$ do there exist isomorphisms $i_{1}: Z \rightarrow X$ and $i_{2}: Z \rightarrow Y$ such that $i_{1} \circ T=i_{2}$.

2. There exists no $\sigma\left(X^{\prime}, X\right)$-closed subspace $M$ of $X^{\prime}$ with $\operatorname{codim}(M)=\infty$ such that $\phi_{M} \circ T^{\prime}$ maps $Y^{\prime}$ into $X^{\prime} / M$.

3. For every closed subspace $M$ of $X$ with $\operatorname{dim}(M)=\infty$, there exists a closed subspace $N$ of $M$ with $\operatorname{dim}(N)=\infty$ such that $T \circ i_{N}$ is a nuclear operator (compact operator). The space $N$ may be constructed to contain any predetermined member of $M$. 
4. For every $\sigma\left(X^{\prime}, X\right)$-closed subspace $M$ of $X^{\prime}$ with $\operatorname{codim}(M)=\infty$, there exists a $\sigma\left(X^{\prime}, X\right)$-closed subspace $N$ containing $M$ with $\operatorname{codim}(N)=\infty$ such that $\phi_{N} \circ T^{\prime}$ is a nuclear operator (compact operator). The space $N$ may be constructed to contain any predetermined member of $X^{\prime}$.

Proof. There is no loss in generality in assuming the subspaces of 1 (a) to be closed. Then $1(\mathrm{~b})$ is just a restatement of $1(\mathrm{a})$.

(1) $\Leftrightarrow(2)$. Suppose the negation of (2) and let $M$ be the offending subspace of $X^{\prime}$. Using the diagram (A), it can be seen that $\left(i_{M_{\perp}}\right)^{\prime} \circ T^{\prime}=\psi_{M_{\perp}} \circ \phi_{M} \circ T^{\prime}$. Since $\psi_{M_{\perp}}$ is an isomorphism with range $M^{\prime}$ the map $\left(i_{M_{\perp}}\right)^{\prime} \circ T^{\prime}$, which is the adjoint of $T \circ i_{M_{\perp}}$, has range $\left(M_{\perp}\right)^{\prime}$. By Theorem II.3.11 [8], $T \circ i_{M_{\perp}}$ is an isomorphism, which proves the negation of $1(\mathrm{a})$. That $(2) \Rightarrow(1)$ is proved similarly.

(1) $\Leftrightarrow(3)$. The proof that (3) $\Rightarrow$ (1) follows from the fact that an isomorphism with infinite-dimensional range cannot be compact. To prove $(1) \Rightarrow(3)$ we modify the proof of Theorem II.1.9 [8] in order to obtain a nuclear operator. Let $x_{0}$ be a predetermined point of $M$ with $\left\|x_{0}\right\|=1$. Use the Hahn-Banach theorem I.5.5 of [8] to obtain an $x_{0}^{\prime}$ in $X^{\prime}$ with $\left\langle x_{0}, x_{0}^{\prime}\right\rangle=\left\|x_{0}^{\prime}\right\|=1$. The kernel of $x_{0}^{\prime}$ is an infinitedimensional subspace of $X$; and since $T$ is strictly singular, it is not invertible on the kernel. There is an element $x_{1}$ in the null space of $x_{0}^{\prime}$ such that $\left\|x_{1}\right\|=1$ and $\left\|T x_{1}\right\|$ $<4^{-1}$ (Theorem I.3.7 of [8]). Employ the Hahn-Banach theorem again to obtain an $x_{1}^{\prime}$ in $X^{\prime}$ with $\left\langle x_{1}, x_{1}^{\prime}\right\rangle=\left\|x_{1}^{\prime}\right\|=1$. We choose the point $x_{2}$ in the infinite-dimensional subspace formed by the intersection of the kernels of $x_{0}^{\prime}$ and $x_{1}^{\prime}$ such that $\left\|x_{2}\right\|=1$ and $\left\|T x_{2}\right\|<4^{-2}$. In a similar manner, we proceed to construct sequences $\left\{x_{i}\right\}$ in $X$ and $\left\{x_{i}^{\prime}\right\}$ in $X^{\prime}$ with the properties:

(1) $\left\|x_{i}\right\|=\left\|x_{i}^{\prime}\right\|=\left\langle x_{i}, x_{i}^{\prime}\right\rangle=1$ for $i=0,1,2, \ldots$,

(2) $\left\|T x_{i}\right\|<4^{-i}, i>0$,

(3) $\left\langle x_{k}, x_{i}^{\prime}\right\rangle=0$ for $i<k$.

We define inductively a new sequence of linear functionals $\left\{z_{i}^{\prime}\right\}$ by $z_{0}^{\prime}=x_{0}^{\prime}$, $z_{n}^{\prime}=x_{n}^{\prime}-\sum_{i=0}^{n-1}\left\langle x_{i}, x_{n}^{\prime}\right\rangle z_{i}^{\prime}$. We have for each $x$ in $N=\operatorname{sp}\left\{x_{i}\right\}$ that $x=\sum_{i=0}^{m} \alpha_{i} x_{i}$ for some integer $m$. An inductive computation shows that $\alpha_{i}=\left\langle x, z_{i}^{\prime}\right\rangle$ for each $i$. It is also seen that $\left\|z_{i}^{\prime}\right\| \leqq 2^{i}$ for each $i$. Define a new sequence of linear functionals $\left\{w_{i}^{\prime}\right\}$ by $w_{i}^{\prime}=2^{-i} z_{i}^{\prime}$. Thus $\left\{w_{i}^{\prime}\right\}$ is a bounded sequence of linear functionals and $x=$ $\sum_{i=0}^{m} 2^{i}\left\langle x, w_{i}^{\prime}\right\rangle x_{i}$ for each $x$ in $N$. We now demonstrate that the sequence of finitedimensional range operators $\sum_{i=0}^{j} 2^{-i}\left\langle-, w_{i}^{\prime}\right\rangle 4^{i} T x_{i}$ converges in norm on $N$ to the operator $T$. In other words, the nuclear operator $\sum_{i=0}^{\infty} 2^{-i}\left\langle-, w_{i}^{\prime}\right\rangle 4^{i} T x_{i}$ and the operator $T$ coincide on $N$. To see this let $\varepsilon>0$ be given and choose $j>0$ such that $\sum_{i=j+1}^{\infty} 2^{-i}<\varepsilon$. Let $x$ be any point in $N$ of norm one and assume $m>j$. Write $x=\sum_{i=0}^{m} 2^{i}\left\langle x, w_{i}^{\prime}\right\rangle x_{i}, T x=\sum_{i=0}^{m} 2^{-i}\left\langle x, w_{i}^{\prime}\right\rangle 4^{i} T x_{i}$. Thus

$$
\left\|T x-\sum_{i=1}^{j} 2^{-i}\left\langle x, w_{i}^{\prime}\right\rangle 4^{i} T x_{i}\right\| \leqq\left\|\sum_{i=j+1}^{m} 2^{-i}\left\langle x, w_{i}^{\prime}\right\rangle 4^{i} T x_{i}\right\| \leqq \sum_{i=j+1}^{\infty} 2^{-i}<\varepsilon .
$$

Both operators are continuous and thus agree on the closure of $N$. 
The restriction of $T$ to $N$ is also compact, because every nuclear operator is compact [17]. Thus if $T$ has a bounded inverse on a subspace of $N$ the subspace must be finite dimensional. This completes the proof of $(1) \Rightarrow(3)$.

(3) $\Leftrightarrow(4)$. To prove (4) from (3), let $M$ be a $\sigma\left(X^{\prime}, X\right)$-closed subspace of $X^{\prime}$ with $\operatorname{codim}(M)=\infty$ and let $x^{\prime}$ be an arbitrary point in $X^{\prime}$. Note that

$$
\operatorname{dim}\left(M_{\perp} \cap \operatorname{ker}\left(x^{\prime}\right)\right)=\infty .
$$

By (3) there exists a closed subspace $N$ of $M_{\perp} \cap \operatorname{ker}\left(x^{\prime}\right)$ such that $T \circ i_{N}$ is a nuclear operator. Since the adjoint of a nuclear operator is nuclear [17, Proposition 47.5], $\left(T \circ i_{N}\right)^{\prime}=i_{N}^{\prime} \circ T^{\prime}=\psi_{N} \circ \phi_{N^{\perp}} \circ T^{\prime}$ is a nuclear operator. This results in a nuclear operator $\phi_{N^{\perp}} \circ T^{\prime}=\psi_{N}^{-1} \circ \psi_{N} \circ \phi_{N^{\perp}} \circ T^{\prime}$, where $N^{\perp}$ is a $\sigma\left(X^{\prime}, X\right)$-closed subspace of $X^{\prime}, N^{\perp} \supset M, x^{\prime} \in N^{\perp}$, and $\operatorname{codim}\left(N^{\perp}\right)=\infty$ [17, Proposition 47.1].

We cannot reverse the above argument to prove (4) $\Rightarrow$ (3) because we cannot prove in general that $T$ is a nuclear operator whenever $T^{\prime}$ is. We can, however, reverse the argument for compact operators; and since (3) concerning a compact operator implies 1(a), this in turn implies the nuclear version of (3).

1.2. TheOREM. Let $T$ be a bounded linear operator mapping the Banach space $X$ into the Banach space $Y$. The following statements are equivalent:

1. (a) The operator $T$ is strictly cosingular, i.e. there is no closed subspace $M$ of $Y$ with $\operatorname{codim}(M)=\infty$, such that $\phi_{M} \circ T$ maps $X$ onto $Y / M$.

(b) The operator $T$ is strictly cosingular (Petczyński [13]), i.e. for no Banach space $Z$ with $\operatorname{dim}(Z)=\infty$ are there continuous surjective operators $h_{1}: X \rightarrow Z$ and $h_{2}: Y \rightarrow Z$ such that $h_{2} \circ T=h_{1}$.

2. There exists no $\sigma\left(X^{\prime}, X\right)$-closed subspace $N$ of $X^{\prime}$ with $\operatorname{dim}(N)=\infty$ such that $i_{N} \circ T^{\prime}$ is an isomorphism (Vladimirskiı [18]).

3. For every $\sigma\left(Y^{\prime}, Y\right)$-closed subspace $M$ of $Y^{\prime}$ with $\operatorname{dim}(M)=\infty$ there exists a $\sigma\left(Y^{\prime}, Y\right)$-closed subspace $N$ of $M$ with $\operatorname{dim}(N)=\infty$ such that $i_{N} \circ T^{\prime}$ is a nuclear operator (compact operator). The space $N$ may be constructed to contain any predetermined member of $M$.

4. For every closed subspace $M$ of $Y$ with $\operatorname{codim}(M)=\infty$, there exists a closed subspace $N$ of $Y$ containing $M$ such that $\operatorname{codim}(N)=\infty$ and $\phi_{N} \circ T: X \rightarrow Y / N$ is a nuclear operator (compact operator). The space $N$ may be constructed to contain any predetermined member of $Y$.

Proof. $1(\mathrm{a}) \Leftrightarrow 1(\mathrm{~b})$. If we assume the negation of 1(b), then $h_{2}$ gives a mapping $\bar{h}_{2}: Y / \operatorname{ker}\left(h_{2}\right) \rightarrow Z$ which is an (onto) isomorphism, so $\phi_{\operatorname{ker}\left(h_{2}\right)} \circ T=\bar{h}_{2}^{1} \circ h_{2} \circ T$ has range $Y / \operatorname{ker}\left(h_{2}\right)$ and codim $\left(\operatorname{ker}\left(h_{2}\right)\right)=\infty$. From the negation of $1(\mathrm{a})$, let $Z=Y / M$, $h_{2}=\phi_{M}$, and $h_{1}=\phi_{M} \circ T$ to obtain the negation of $1(\mathrm{~b})$.

(1) $\Leftrightarrow(2)$. Suppose the negation of 1(a) and let $M$ be the closed subspace of $Y$ such that $\phi_{M} \circ T$ is onto. By Theorem II.3.13 of [8], $T^{\prime} \circ \phi_{M}^{\prime}$ is an isomorphism. But $\phi_{M}^{\prime}:(Y / M)^{\prime} \rightarrow M^{\perp}$ is also a surjective isomorphism. It follows that $T^{\prime} \circ i_{M^{\perp}}$ is an isomorphism. 
For the converse assume that there is a $\sigma\left(Y^{\prime}, Y\right)$-closed subspace $M$ of $Y^{\prime}$ with $\operatorname{codim}(M)=\infty$ and $T^{\prime} \circ i_{M}$ an isomorphism. The adjoint of $\phi_{M_{\perp}} \circ T$ is $T^{\prime} \circ \phi_{M_{\perp}}^{\prime}$. The map $\phi_{M_{\perp}}^{\prime}$ is an isomorphism of $\left(Y / M_{\perp}\right)^{\prime}$ onto $\left(M_{\perp}\right)^{\perp}=M$. Thus $T^{\prime} \circ \phi_{M_{\perp}}^{\prime}$ is an isomorphism. By [6, Lemma VI.6.3], $\phi_{M_{\perp}} \circ T$ is onto.

(2) $\Leftrightarrow(3)$. The proof of (2) $\Rightarrow(3)$ is similar to the proof of (1) $\Rightarrow(3)$ in Theorem 1.1, except that we are now unable to make use of the Hahn-Banach theorem for the norm topology on the dual space because the linear functionals must be in the original space $Y$ in order to continuously extend our operators over the $\sigma\left(Y^{\prime}, Y\right)$ closure of a subspace of $Y^{\prime}$. Commence the proof by considering an infinitedimensional $\sigma\left(Y^{\prime}, Y\right)$-closed subspace $M$ of $Y^{\prime}$. We now construct a $\sigma\left(Y^{\prime}, Y\right)$ closed subspace $N$ of $M$ such that the restriction of $T$ to $N$ is a nuclear operator. Let $y_{0}^{\prime}$ be a predetermined member of $M$ with $\left\|y_{0}^{\prime}\right\|=1$.

Since $\left\|y_{0}^{\prime}\right\|=\sup \left\{\left\langle y, y_{0}^{\prime}\right\rangle:\|y\|=1\right\}$, there exists $y_{0}$ in $Y$ with $\left\|y_{0}\right\|=1$ and $\frac{1}{2} \leqq\left\langle y_{0}, y_{0}^{\prime}\right\rangle \leqq 1$. The space $R=\operatorname{sp}\left[y_{0}\right]$ has infinite codimension in $Y$. Thus $R^{\perp}$ is a $\sigma\left(Y^{\prime}, Y\right)$-closed subspace of $Y^{\prime}$ with $\operatorname{dim}\left(R^{\perp}\right)=\infty$. Since $T^{\prime}$ is not invertible on $R^{\perp}$, there exists $y_{1}^{\prime}$ in $R^{\perp}$ with $\left\|y_{1}^{\prime}\right\|=1$ and $\left\|T^{\prime} y_{1}^{\prime}\right\| \leqq\left(\frac{1}{2}\right) 6^{-1}$. We choose the point $y_{1}$ in $Y$ such that $\left\|y_{1}\right\|=1$ and $\frac{1}{2} \leqq\left\langle y_{1}, y_{1}^{\prime}\right\rangle \leqq 1$.

If we denote by $R$ the $\mathrm{sp}\left[y_{0}, y_{1}\right]$, there exists $y_{2}^{\prime}$ in $R^{\perp}$ such that $\left\|y_{2}^{\prime}\right\|=1$ and $\left\|T^{\prime} y_{2}^{\prime}\right\| \leqq\left(\frac{1}{2}\right) 6^{-2}$. In a similar manner, by induction we establish sequences $\left\{y_{i}^{\prime}\right\}$ in $Y^{\prime}$ and $\left\{y_{i}\right\}$ in $Y$ with the properties:

(1) $\left\|y_{i}\right\|=\left\|y_{i}^{\prime}\right\|=1$ for $i=0,1,2, \ldots$,

(2) $\left\|T^{\prime} y_{i}^{\prime}\right\| \leqq\left(\frac{1}{2}\right) 6^{-i}, i>0$,

(3) $\left\langle y_{i}, y_{k}^{\prime}\right\rangle=0$ for $i<k$,

(4) $\frac{1}{2} \leqq\left\langle y_{i}, y_{i}^{\prime}\right\rangle \leqq 1$ for all $i$.

Let $\beta_{i}=1 /\left\langle y_{i}, y_{i}^{\prime}\right\rangle$. Then $1 \leqq \beta_{i} \leqq 2$ for all $i$. Inductively we define the sequence $\left\{w_{k}\right\}$ of elements of $Y$ by $w_{0}=\beta_{0} y_{0}$, and $w_{n}=\beta_{n}\left[y_{n}-\sum_{i=0}^{n-1}\left\langle y_{n}, y_{i}^{\prime}\right\rangle w_{i}\right]$. If $W$ denotes $\operatorname{sp}\left[\left\{y_{i}^{\prime}\right\}\right]$, we can express each $y^{\prime}$ in $W$ as $y^{\prime}=\sum_{i=0}^{m} \alpha_{i} y_{i}^{\prime}$. An inductive computation shows that $\alpha_{i}=\left\langle w_{i}, y_{i}^{\prime}\right\rangle, i=0, \ldots, m$. It is also seen that $\left\|w_{i}\right\| \leqq 2 \cdot 3^{i}$.

We replace the sequence $\left\{w_{i}\right\}$ by a sequence $\left\{u_{i}\right\}$ by letting $u_{i}=w_{i} /\left(2 \cdot 3^{i}\right)$. Thus $\left\|u_{i}\right\|=1$. Let $x_{i}^{\prime}=T^{\prime}\left(y_{i}^{\prime}\right) 2 \cdot 6^{i}$ for $i=0,1,2, \ldots$ We now have a nuclear operator $\sum_{i=0}^{\infty}\left(1 / 2^{i}\right)\left\langle u_{i},->x_{i}^{\prime}\right.$. The same argument as used in the corresponding place in the proof of Theorem 1.1 shows that this nuclear operator coincides with $T^{\prime}$ on $W$. Our nuclear operator is the transpose of the operator $\sum_{i=0}^{\infty}\left(1 / 2^{i}\right)\left\langle-, x_{i}^{\prime}\right\rangle u_{i}$ and thus our nuclear operator is continuous for the $\sigma\left(Y^{\prime}, Y\right)$ - and $\sigma\left(X^{\prime}, X\right)$-topologies on $Y^{\prime}$ and $X^{\prime}$ respectively. Since $T^{\prime}$ is also continuous for these topologies, the nuclear operator and $T^{\prime}$ will coincide on the $\sigma\left(Y^{\prime}, Y\right)$-closure of $W$, call it $N$. This proves (2) $\Rightarrow$ (3).

Because a nuclear operator is compact we use the same type of argument as found in Theorem $1.1((3) \Rightarrow(1))$ to prove (3) $\Rightarrow(2)$.

(3) $\Leftrightarrow(4)$. We have to be careful in proving (3) $\Rightarrow(4)$ because we do not know if an operator is nuclear when its adjoint is nuclear. Let $M$ be a closed subspace of $Y$ with $\operatorname{codim}(M)=\infty$, and let $y_{0}$ be an arbitrary point in $Y$. Let $Z=\operatorname{sp}\left[M \cup\left\{y_{0}\right\}\right]$. The space $Z^{\perp}$ is a $\sigma\left(Y^{\prime}, Y\right)$-closed subspace of $Y^{\prime}$ and $\operatorname{dim}\left(Z^{\perp}\right)=\infty$. Since (3) is 
equivalent to (2), we can repeat the construction in the proof of (3) to obtain a nuclear operator $K^{\prime}$, which is the adjoint of a nuclear operator $K$, and which agrees with $T^{\prime}$ on an infinite-dimensional subspace $W$ of $Z^{\perp}$. Let $R=\operatorname{ker}\left(K^{\prime}-T^{\prime}\right) \cap Z^{\perp}$; and note that $R$ is $\sigma\left(Y^{\prime}, Y\right)$-closed and $\operatorname{dim}(R)=\infty$. Then $N=R_{\perp}$ is the desired subspace of $Y$. Note that $y_{0} \in N \supseteq M$, and since $\left(\phi_{N} \circ T\right)^{\prime}=T^{\prime} \circ \phi_{N}^{\prime}=K^{\prime} \circ \phi_{N}^{\prime}$ $=\left(\phi_{N} \circ K\right)^{\prime}, \phi_{N} \circ T$ agrees with the nuclear operator $\phi_{N} \circ K[17]$.

There is no problem in going from (4) to (3) because the adjoint of a nuclear operator is a nuclear operator.

1.3. REMARK. The characterizations of strictly singular and strictly cosingular operators given in Theorems 1.1 and 1.2 point out the relationships between the operators and their adjoint operators. The relationships hinge on the $\sigma\left(X^{\prime}, X\right)$ closed and $\sigma\left(X^{\prime}, X^{\prime \prime}\right)$-closed subspaces of $X^{\prime}$ and the corresponding subspaces of $Y^{\prime}$. A variation of Theorem 1.1 can be stated for normed linear spaces because $X^{\prime}$ and $Y^{\prime}$ are Banach spaces. Theorem 1.2 must be stated for Banach spaces. Pelczyński [13] has noted that if the adjoint of an operator is strictly singular (strictly cosingular), the operator is strictly cosingular (strictly singular); and when $X$ is reflexive ( $Y$ is reflexive), the adjoint of a strictly singular (strictly cosingular) operator is strictly cosingular (strictly singular). These results can be verified by examining the statements of the above theorems.

1.4. Corollary. If $T$ is a compact operator mapping the Banach space $X$ into the Banach space $Y$ then

(a) for every closed infinite-dimensional subspace $M$ of $X$ there is a closed infinitedimensional subspace $N \subset M$ such that $T$ restricted to $N$ is a nuclear operator. The space $N$ may be taken to contain any prescribed member of $M$,

(b) for every closed subspace $U$ of $Y$, codim $(U)=\infty$, there is a closed subspace $V$ of $Y, V \supset U, \operatorname{codim}(V)=\infty$, such that $\phi_{V} \circ T: X \rightarrow Y / V$ is a nuclear operator. The space $V$ may be taken to contain any prescribed member of $Y$.

Proof. Every compact operator is both strictly singular and strictly cosingular.

2. Approximation convergence structures. In Theorem 1.1(3) a strictly singular operator is characterized by the property that its restrictions to certain subspaces are compact operators. This property may be used to employ knowledge of approximating compact operators by finite-dimensional range operators ([2], [4]) to the problem of approximating strictly singular operators. In fact we will see that any given strictly singular operator $T$ may be approximated by finite-dimensional range operators using a locally convex Hausdorff topology on the space $\mathscr{L}[X, Y]$ of all bounded linear operators with domain $X$ and range in $Y$. The convergence structures arise when we attempt to encompass all the strictly singular operators. Although our techniques work when $X$ and $Y$ are not complete, we assume for convenience that $X$ and $Y$ are Banach spaces.

For the fixed strictly singular operator $T$, let $\mathscr{S}_{T}$ denote the collection of unit balls from the closed infinite-dimensional subspaces to which the restrictions of $T$ result 
in compact operators. From Theorem 1.1, the collection $\mathscr{S}_{T}$ has the following properties:

(1) $\mathscr{S}_{T}$ is nonempty.

(2) For every closed infinite-dimensional subspace $M$ of $X$ and for every $x$ in $M$ with $\|x\| \leqq 1$, there exists an $S$ in $\mathscr{S}_{T}$ such that $x \in S \subset M$.

(3) If $S_{1} \in \mathscr{S}_{T}$ and $S_{2}$ is the unit ball of a closed infinite-dimensional subspace such that $S_{2} \subset S_{1}$, then $S_{2} \in \mathscr{S}_{T}$.

Next we want to define convergence topologies using the family $\mathscr{S}_{T}$, but prior to doing so it is desirable to generalize our setting so that we will not be tied to a single operator. Let $\subseteq$ be the class whose elements are all the collections of unit balls of closed infinite-dimensional subspaces, where each such collection must satisfy the above-mentioned properties (1), (2), and (3) that $\mathscr{S}_{T}$ satisfy. For any $\mathscr{S}$ in $\Xi$ there corresponds a collection of strictly singular operators, namely those whose restrictions to precisely those closed infinite-dimensional subspaces with unit ball in $\mathscr{S}$ are compact. Further, for every strictly singular $T, \mathscr{S}_{T} \in \mathbb{S}$.

Each collection $\mathscr{S}$ in $\mathfrak{S}$ can be used to define locally convex Hausdorff topologies on $\mathscr{L}[X, Y]$. Either uniform convergence over $\mathscr{S}$ or almost uniform convergence over $\mathscr{S}$ ([1], [2], [3], [4]) may be used, depending on what one is willing to assume about $Y$. Let us digress for a moment and consider the simplified situation where $\mathscr{S}$ consists of a single element, the unit ball $S_{X}$ of $X$ (the associated operators'are the compact operators). For the topology of almost uniform convergence on $S_{X}$, we obtain the compact operators as the closure of the bounded operators of finitedimensional range ([2], [4]). The space $Y$ having the approximation property means that the topology of uniform convergence may be used. (It is an unsolved problem whether every Banach space has the approximation property.) Looking again at the general collection $\mathscr{S}$, it is shown in [4] that the closure in $\mathscr{L}[X, Y]$ of the finitedimensional range operators under either of these topologies consists of those operators which map the members of $\mathscr{S}$ into compact sets, in other words those operators whose restrictions to subspaces spanned by members of $\mathscr{S}$ are compact operators. Theorem 1.1 tells us then that all the operators in this closure are strictly singular operators.

There are other topologies which may be defined by using $\mathscr{S}$ to play the desired role for strictly singular operators. Specifically, we will consider the following:

(A) The topology of almost uniform convergence on members of $\mathscr{S}$ with the norm topology on $Y$.

(B) View the adjoints of all bounded linear operators and use the topology of almost uniform convergence on the unit ball of $Y^{\prime}$ with $X^{\prime}$ having the topology of uniform convergence on members of $\mathscr{S}$.

(C) The supremum of topologies (A) and (B).

(D) Assume $Y$ has the approximation property. Then we use the topology of uniform convergence on members of $\mathscr{S}$ with the norm topology on $Y$.

Systematic treatment of such topologies appears in [4]. 
An operator $T: X \rightarrow Y$ defines a linear form on the tensor product $X \otimes Y^{\prime}$ by mapping $x \otimes y^{\prime}$ to $\left\langle T x, y^{\prime}\right\rangle$. Elements of $X^{\prime} \otimes Y$ may be viewed as linear forms on $X \otimes Y^{\prime}$ and these linear forms correspond to the bounded finite-dimensional range operators mapping $X$ into $Y$. If $A \subset X$ and $B \subset Y^{\prime}$, then $A \otimes B=\{a \otimes b: a \in A$, $b \in B\}$.

2.1. ThEOREM. A bounded linear operator $T$ is strictly singular if and only if there exists an $\mathscr{S} \in \cong$ such that $T$ is in the closure of the bounded linear operators of finitedimensional range for the topology $\tau$, where $\tau$ is related to $\mathscr{S}$ by any of the topologies of type (A), (B), or (C) listed above. If $Y$ has the approximation property the topology of type (D) may be used.

Proof. Let $T$ be a strictly singular operator. Theorem 1.1(3) gives us the associated collection $\mathscr{S}_{T}$ from $\mathfrak{S}$. Denote by $\tilde{\mathscr{S}}_{T}$ the collection of closed convex hulls of finite unions of members of $\mathscr{S}_{T}$. The operator $T$ maps each member of $\tilde{\mathscr{S}}_{T}$ into a compact set [10, Proposition 3.5.2]. Let $S^{\prime}$ be the unit ball of $Y^{\prime}$ and let $A$ be a member of $\tilde{\mathscr{S}}_{T}$. Lemma $2.1((2) \Rightarrow(3))$ from [4] tells us that the restriction of the linear form $T$ to $A \otimes S^{\prime}$ is continuous for the $\sigma\left(X \otimes Y^{\prime}, X^{\prime} \otimes Y\right)$-topology. The linear form $T$ is in the closure of $X^{\prime} \otimes Y$ for the topology of almost uniform convergence on members of $\left\{A \otimes S^{\prime}: A \in \tilde{\mathscr{S}}_{T}\right\}$ [4, Theorem 1.4]. This topology is the weak topology for the topologies (A), (B), and (C) on the closure of $X^{\prime} \otimes Y$ [4, Theorem 3.3]. Since the closure of a convex set is unchanged for compatible topologies [10, Proposition 3.4.3], we see that the strictly singular operator $T$ is in the closure of the bounded linear operators of finite-dimensional range for the three topologies.

When $Y$ has the approximation property the linear form $T$ is $\sigma\left(X \otimes Y^{\prime}, X^{\prime} \otimes Y\right)$ continuous when restricted to the convex hull of $A \otimes S^{\prime}$ for every $A \in \tilde{\mathscr{S}}_{T}$ [4, Theorem 4.8(e)]. A modification of Grothendieck's completion theorem [4, Theorem 1.1] tells us that $T$ is in the completion of $X^{\prime} \otimes Y$ for the topology of uniform convergence on the set $\left\{A \otimes S^{\prime}: A \in \tilde{\mathscr{S}}_{T}\right\}$. For $\mathscr{L}[X, Y]$ this is the topology (D).

For the converse, suppose $T$ is in the closure of $X^{\prime} \otimes Y$ for the selected topology. Then $T$ is in the closure of $X^{\prime} \otimes Y$ for the topology of almost uniform convergence on members of $\left\{A \otimes S^{\prime}: A \in \tilde{\mathscr{S}}\right\}$ [4, Theorem 3.3]. Thus, $T$ has $\sigma\left(X \otimes Y^{\prime}, X^{\prime} \otimes Y\right)-$ continuous restrictions to $A \otimes S^{\prime}$ for every $A \in \tilde{\mathscr{S}}$. From Lemma 2.1 of [4], $T(A)$ is totally bounded for every $A \in \tilde{\mathscr{S}}$. By property (2) of the family $\mathscr{S}, T$ satisfies condition (3) of Theorem 1.1.

We have taken one step in the direction of characterizing the strictly singular operators as the closure of continuous finite-dimensional range operators; but when one pieces together the collection of topologies which are generated as $\mathscr{S}$ ranges through $\subseteq$, the resultant convergence process is nontopological. Thus it is useful to bring in the concept of a convergence structure.

2.2. Definition. Let $G$ be any set and let $\gamma$ be a map from $G$ into the power set 
of all filters on $G$ (i.e. $\gamma x$ is a collection of filters on $G$ for each $x$ in $G$ ). Then $\gamma$ is a convergence structure [7] on $G$ if

(1) For each $x$ in $G, \gamma x$ is a $\wedge$-ideal, i.e. filters $\mathscr{F}$ and $\mathscr{G}$ are in $\gamma x$ if and only if $\mathscr{F} \wedge \mathscr{G}$ (the filter having as a base $\{F \cup G: F \in \mathscr{F}, G \in \mathscr{G}\}$ ) is in $\gamma x$.

(2) For each $x$ in $G$, the filter of all sets containing $x$ is in $\gamma x$.

The convergence structure is Hausdorff if $\gamma x \cap \gamma y=\varnothing$ whenever $x \neq y$.

The convergence structure $\gamma$ is linear if

(1) For $\mathscr{F} \in \gamma x$ and $\mathscr{G} \in \gamma y$ it follows that $\mathscr{F}+\mathscr{G} \in \gamma(x+y)$.

(2) For a filter $\mathscr{K}$ converging to scalar $\alpha$ and $\mathscr{F} \in \gamma x$ it follows that $\mathscr{K} \cdot \mathscr{F} \in \gamma(\alpha x)$.

Some additional notation will be helpful. Select one of the topologies (A), (B), (C), or (D). Let $J$ be an index set for the class $\mathbb{S}$, and for a member $\alpha$ of $J$ let $\tau_{\alpha}$ be the topology corresponding to the collection of unit balls $\mathscr{S}_{\alpha}$. Define a partial order $>$ on $J$ as follows: For $\alpha, \beta \in J, \alpha>\beta$ if and only if $\mathscr{S}_{\alpha} \subset \mathscr{S}_{\beta}$, which is true if and only if $\tau_{\alpha}$ is weaker than $\tau_{\beta}$.

2.3. Lemma. $(J,>)$ is a directed set.

Proof. For $\alpha, \beta$ in $J$, let the family $\mathscr{S}_{\gamma}$ be defined by $\mathscr{S}_{\alpha} \cap \mathscr{S}_{\beta}$. This family has properties (1) and (3) required for membership in $\subseteq$. But property (3) for $\mathscr{S}_{\beta}$ can be used to show that $\mathscr{S}_{\gamma}$ has property (2). Thus $\mathscr{S}_{\gamma} \in \subseteq$ and $\gamma>\alpha, \gamma>\beta$.

We are now in a position to define our convergence structure on $\mathscr{L}[X, Y]$.

2.4. Definition. For $T \in \mathscr{L}[X, Y], \gamma(T)$ is defined as all filters on $\mathscr{L}[X, Y]$ which converge to $T$ for at least one $\tau_{\alpha_{0}}, \alpha_{0} \in J$. The element $T$ is a $\gamma$-limit point of the subset $A$ of $\mathscr{L}[X, Y]$ if and only if there is a filter $\mathscr{F}$ in $\gamma(T)$ such that $A \in \mathscr{F}$.

Since $\tau_{\alpha}$ is weaker than $\tau_{\alpha_{0}}$ when $\alpha>\alpha_{0}$, any filter which converges for $\tau_{\alpha_{0}}$ also converges for $\tau_{\alpha}, \alpha>\alpha_{0}$.

2.5. LEMMA. The map $\gamma$ is a Hausdorff linear convergence structure on $\mathscr{L}[X, Y]$.

Proof. The details are easily verified in light of the fact that $J$ is a directed set.

2.6. THEOREM. With the convergence structure $\gamma$ on $\mathscr{L}(X, Y)$, the strictly singular operators form precisely the set of all limit points of the continuous operators of finite-dimensional range.

Proof. Use Theorem 2.1 and the definitions.

2.7. THEOREM. Assume $Y$ has the approximation property and that the topologies are the type (D). Then the strictly singular operators are the $\gamma$-completion of the bounded operators of finite-dimensional range, i.e. every $\gamma$-Cauchy filter on the space of bounded operators of finite-dimensional range converges to a strictly singular operator and every strictly singular operator is a limit point of such a filter $\left({ }^{2}\right)$.

2.8. The strictly cosingular operators. A completely analogous treatment of strictly cosingular operators can be made based on the characterization from statement (3) of Theorem 1.2. In this case, the collection $\subseteq$ would be composed of unit

$\left(^{2}\right)$ The hypothesis that $Y$ is a Banach space is required for this result. 
balls of $\sigma\left(Y^{\prime}, Y\right)$-closed subspaces of $Y^{\prime}$. The collection $\subseteq$ would have the same three properties as before except that all closed subspaces must now be interpreted as being $\sigma\left(Y^{\prime}, Y\right)$-closed.

2.9. Further generalizations. In reference [9], R. H. Herman discussed operators with "property R", originally suggested by R. J. Whitley. The characterization of such operators differs from statement (3) of Theorem 1.1 only in that weakly compact operators replace the compact operators and nuclear operators. Using tools in [4], it is possible to do a completely analogous treatment for these operators. In fact, statement (3) of Theorem 1.1 or statement (3) of Theorem 1.2 could be used to define numerous classes of operators by replacing the compact and nuclear operators by any of the classes of operators whose approximation topologies are given in reference [4]. There you will find treatments of the linear operators, continuous linear operators, weakly compact operators, completely continuous operators, and the compact operators.

3. Approximation using nonstandard locally convex spaces. In the previous section we obtained an approximation convergence structure on $\mathscr{L}[X, Y]$ rather than a topology. This is disappointing in that we cannot immediately utilize the theory of locally convex linear topological spaces. This disadvantage can be overcome by formulating an extension of $\mathscr{L}[X, Y]$ in the manner of nonstandard analysis [14]. This extension is a nonstandard model of a locally convex space. A great deal of the standard locally convex theory carries over to the nonstandard space under the proper interpretations. An example is seen in the nonstandard Hahn-Banach Theorem 3.9. Since our discussion at this time does not depend on $\mathscr{L}[X, Y]$ being a space of operators, let us replace $\mathscr{L}[X, Y]$ by an abstract linear space $Z$ with analogous locally convex topologies and convergence structures.

3.1. Definition. A directed family $\left\{\tau_{\alpha}: \alpha \in J\right\}$ of Hausdorff locally convex topologies on $Z$ is a collection of topologies indexed over a directed set $J$ with the property that, for $\alpha$ and $\beta$ in $J, \alpha>\beta$ if and only if $\tau_{\alpha}$ is weaker than $\tau_{\beta}$. The associated convergence structure $\gamma$ will be determined as in Definition 2.4.

We now proceed with the construction of the nonstandard extension. A general discussion can be found in Chapters 2 and 4 of [14]. For each $\alpha$ in $J$ let $Z_{\alpha}$ be $Z$ with the topology $\tau_{\alpha}$. Consider the product set $Z^{J}=\prod\left\{Z_{\alpha}: \alpha \in J\right\}$ on which we place the box topology [12, p. 107]. This topology does not make $Z^{J}$ a linear topological space, but we do have continuity of addition (coordinatewise). Similarly for the scalar field $K$ (the real or complex numbers) obtain $K^{J}=\prod\left\{K_{\alpha}: \alpha \in J\right\}$ with the box topology. For each $\alpha$ in $J, K_{\alpha}$ has the usual topology of $K$. Multiplication and addition are defined coordinatewise.

In order to obtain the nonstandard model, an equivalence relation on $Z^{J}$ and $K^{J}$ is defined by means of an ultrafilter. Let $\mathscr{U}$ be an ultrafilter which is a refinement of a filter having as a base sets of the form $\left\{\alpha: \alpha \in J, \alpha>\alpha_{0}\right\}$, where $\alpha_{0}$ ranges through $J$. The equivalence relation $R$ is defined by $u R v$ if and only if $\{\alpha: \alpha \in J$, 
$u(\alpha)=v(\alpha)\}$ is a member of $\mathscr{U}$. Note that $Z_{0}=\left\{u: u \in Z^{J}, u R 0\right\}$ is closed under addition and multiplication by members of $K^{J}$. The set $K_{0}=\left\{\lambda: \lambda \in K^{J}, \lambda R 0\right\}$ is closed under multiplication and addition. Both $Z_{0}$ and $K_{0}$ are closed for the box topologies. We find ourselves in a situation analogous to the development of the quotient space of a linear topological space [10, pp. 37 and 104]. The definition of the nonstandard extension can now be given.

3.2. Definition. The nonstandard model of $Z$ relative to the directed family of topologies $\left\{\tau_{\alpha}: \alpha \in J\right\}$ and the ultrafilter $\mathscr{U}$ is the quotient space $Z^{J} / Z_{0}$ with the quotient topology. It is denoted by ${ }^{*} Z$. The nonstandard model of $K$ is $K^{J} / K_{0}$, denoted by ${ }^{*} K$. It is called the nonstandard scalar field. Addition in ${ }^{*} Z$ and ${ }^{*} K$ is defined as in the quotient of a vector space. For ${ }^{*} u$ in ${ }^{*} Z$ and ${ }^{*} \lambda$ in ${ }^{*} K,{ }^{*} \lambda^{*} u$ is $\left\{\lambda u: \lambda \in{ }^{*} \lambda \subset K^{J}, u \in{ }^{*} u \subset Z^{J}\right\}$. In the same fashion we obtain multiplication in ${ }^{*} K$. (Note that the algebraic operations make ${ }^{*} K$ a field (see [14], [15] for a detailed discussion). We do not follow their convention of denoting only standard points of $* Z$ by $* u$. The symbol $* u$ will denote any member of $* Z$. The analogous notation is also used for members of ${ }^{*} K$.)

3.3. RemarK. The present approach is a slight extension of the usual technique of nonstandard topology (e.g. Chapter 4 of [14]) in that the topologies on $Z$ are different for distinct members of the indexing set $J$. The topology on ${ }^{*} K$ on the other hand is identical to that considered by Robinson.

We must generalize some of the definitions from locally convex space theory before going on.

3.4. Definition. Let $A$ be a subset of a nonstandard model ${ }^{*} Z$ of a linear space $Z$.

(a) The set $A$ is balanced if for every ${ }^{*} z$ in $A$, then ${ }^{*} \lambda^{*} z$ is in $A$ for all ${ }^{*} \lambda$ in ${ }^{*} K$ such that $\left|{ }^{*} \lambda\right| \leqq 1$ (i.e. for each $\lambda$ in the coset ${ }^{*} \lambda \subset K^{J}$ the set $\{\alpha: \alpha \in J,|\lambda(\alpha)| \leqq 1\}$ is a member of $\mathscr{U}$ ).

(b) The set $A$ is absorbing if for every ${ }^{*} z$ in ${ }^{*} Z$ there exists ${ }^{*} \lambda_{0}$ in ${ }^{*} K$ such that ${ }^{*} \lambda^{*} z \in A$, for all $\left.\right|^{*} \lambda \mid \leqq{ }^{*} \lambda_{0}$ (i.e. for each $\lambda \in{ }^{*} \lambda$ and $\lambda_{0} \in{ }^{*} \lambda_{0},\{\alpha: \alpha \in J,|\lambda(\alpha)|$ $\left.\left.\leqq \lambda_{0}(\alpha)\right\} \in \mathscr{U}\right)$.

(c) The set $A$ is convex if, for every ${ }^{*} z_{1}$ and ${ }^{*} z_{2}$ in $A,{ }^{*} \lambda_{1}{ }^{*} z_{1}+{ }^{*} \lambda_{2}{ }^{*} z_{2}$ is in $A$ for all ${ }^{*} \lambda_{1}$ and ${ }^{*} \lambda_{2}$ in $* K$ such that $0 \leqq * \lambda_{1}, 0 \leqq * \lambda_{2}$, and ${ }^{*} \lambda_{1}+{ }^{*} \lambda_{2}=1$ (i.e. for $\lambda_{1}$ from the coset ${ }^{*} \lambda_{1}$ and $\lambda_{2}$ from the coset ${ }^{*} \lambda_{2}$ the three sets, $\left\{\alpha: \alpha \in J, 0 \leqq \lambda_{1}(\alpha)\right\}$, $\left\{\alpha: \alpha \in J, 0 \leqq \lambda_{2}(\alpha)\right\}$, and $\left\{\alpha: \alpha \in J, \lambda_{1}(\alpha)+\lambda_{2}(\alpha)=1\right\}$, are in $\left.\mathscr{U}\right)$.

3.5. THEOREM. Let $Z$ be a linear space with a directed family of Hausdorff locally convex topologies. Then the nonstandard model $* Z$ of $Z$ is a nonstandard locally convex space in the sense that addition and scalar multiplication by members of ${ }^{*} K$ are continuous, and there exists a fundamental system of nonstandard convex, balanced and absorbing neighborhoods of 0 . The topology on ${ }^{*} Z$ is Hausdorff.

Proof. The proof that the quotient map is open, addition is continuous, and that the topology is Hausdorff is identical to the linear space situation [10, pp. 104 and 
105]. Thus we have a fundamental system of neighborhoods of 0 by considering the quotient map images of the sets of the form $\prod\left\{U_{\alpha}: \alpha \in J\right\}$. We take each $U_{\alpha}$ to be a convex balanced neighborhood of 0 in $Z_{\alpha}$. Let $* U$ denote the image of $\prod\left\{U_{\alpha}: \alpha \in J\right\}$. That ${ }^{*} U$ is balanced, convex and absorbing in the nonstandard sense is a straightforward exercise.

Now that we have the fundamental system of neighborhoods, the continuity of scalar multiplication can be proved as on p. 82 of [10].

3.6. Notation and conventions. We have referred to ${ }^{*} Z$ and ${ }^{*} K$ as extensions of $Z$ and $K$ because of the natural injections which are available. An element $z$ of $Z$ is mapped to the coset ${ }^{*} z$ containing the element $Z^{J}$ which is $z$ in every coordinate: In the same way we map $K$ into ${ }^{*} K$. The injections preserve the algebraic operations but are not continuous. Henceforth we will speak of $Z$ and $K$ as subsets of their nonstandard models without mention of the injections. The set $Z$ is called the standard points of $* Z$.

For each subset $A$ of $Z$ we will denote by $A$ the subset of ${ }^{*} Z$ which is the quotient map image of $\Pi\left\{A_{\alpha}: \alpha \in J\right\}$, where $A_{\alpha}=A$ for all $\alpha$. Observe that $* A \cap Z=A$.

For each filter $\mathscr{F}$ on $Z$ we denote by $* \mathscr{F}$ a filter on $* Z$ having a base composed of the quotient map images of sets of the form $\prod\left\{F_{\alpha}: \alpha \in J\right\}$ where each $F_{\alpha}$ ranges through $\mathscr{F}$.

3.7. THEOREM. Let $Z$ be a linear space with a directed family of Hausdorff locally convex topologies and a resulting convergence structure $\gamma$ as in Theorem 2.5. Let $* Z$ be the associated nonstandard locally convex space.

(i) On $Z$ a filter $\mathscr{F}$ belongs to $\gamma(z)$ if and only if $* \mathscr{F}$ converges to $z$ in $* Z$.

(ii) $A$ subset $A$ of $Z$ is open for the final topology (i.e. the finest topology on $Z$ such that all the identity maps from the $Z_{\alpha}$ 's to $Z$ are continuous $\left.[10, p .47]\right)$ if and only if $* A$ is open in $* Z$.

(iii) Let $A$ be a subset of $Z$. $A$ subset $B$ of $Z$ is all the $\gamma$-limit points of $A$ if and only if $Z \cap * \bar{A}=B$, i.e. $B$ is all standard points in the closure of $* A$.

Proof. Statement (i). Suppose $\mathscr{F}$ belongs to $\gamma(z)$. Because of the definition of $\gamma$ and the construction of the ultrafilter $\mathscr{U}$, there is a $D \in \mathscr{U}$ such that, for each $\alpha$ in $D$, the filter $\mathscr{F}$ converges to $z$ for the topology $\tau_{\alpha}$. It suffices to consider a neighborhood * $U$ of 0 which is the quotient map image of $\prod\left\{U_{\alpha}: \alpha \in J\right\}$. For each $\alpha$ in $D$ there is an $F_{\alpha} \in \mathscr{F}$ such that $F_{\alpha} \subset z+U_{\alpha}$. Take $F_{\alpha}=Z$ for $\alpha \notin D$. Let ${ }^{*} F$ be the quotient map image of $\prod\left\{F_{\alpha}: \alpha \in J\right\}$. The set ${ }^{*} F$ belongs to $* \mathscr{F}$ and we must show that it a subset of $z+{ }^{*} U$. Consider an arbitrary ${ }^{*} u \in{ }^{*} F$ and a $u$ from the coset ${ }^{*} u$. Then the set $\left\{\alpha: u(\alpha) \in F_{\alpha}\right\}$ belongs to $\mathscr{U}$. For the same $\alpha$ 's, $u(\alpha)$ will be in $Z+U_{\alpha}$. Thus ${ }^{*} u \in{ }^{*} z+{ }^{*} U$.

For the converse suppose $\mathscr{F}$ does not belong to $\gamma(z)$. Thus there is a $D$ in $\mathscr{U}$ such that for each $\alpha$ in $D$ the filter $\mathscr{F}$ does not converge to $z$ for the topology $\tau_{\alpha}$. For each of these $\alpha$ 's there is a neighborhood $z+U_{\alpha}$ which does not contain a member 
of $\mathscr{F}$. Let ${ }^{*} U$ be the quotient mapimage of $\prod\left\{U_{\alpha}: \alpha \in J\right\}$ where we take $U_{\alpha}=Z$ for $\alpha \notin D$. We consider from * an arbitrary set ${ }^{*} F$ which is the image of $\prod\left\{F_{\alpha}: \alpha \in J\right\}$. For each $\alpha \in D$ there is a $u(\alpha) \in F_{\alpha}$ such that $u(\alpha) \notin U_{\alpha}$. Let ${ }^{*} u$ be the coset containing such functions. Observe that ${ }^{*} u \in{ }^{*} F$ but ${ }^{*} u \notin z+{ }^{*} U$. This contradicts the convergence to $z$ of $* \mathscr{F}$.

The other assertions yield to the same type of argument.

In preparation for a nonstandard Hahn-Banach theorem we will want to single out a special class of linear forms defined on $* Z$.

3.8. Definition. Let $\left\{f_{\alpha}: \alpha \in J\right\}$ be a family of linear forms on $Z$ such that each $f_{\alpha}$ is continuous for the topology $\tau_{\alpha}$. Define a map ${ }^{*} f$ of ${ }^{*} Z$ into ${ }^{*} K$ by ${ }^{*} f\left({ }^{*} z\right)$ $=\left\{k \in K^{J}:\left\{\alpha: k(\alpha)=f_{\alpha}(z(\alpha))\right\} \in \mathscr{U}\right.$ for all $z$ from the coset $\left.*_{z}\right\}$. Such maps are called admissible linear functionals. (The reader may verify that they are ${ }^{*} K$ linear and continuous.)

3.9. THEOREM. Let $Z$ and ${ }^{*} Z$ be as in Theorem 3.7. Let ${ }^{*} M$ be the quotient map image of $\Pi\left\{M_{\alpha}: \alpha \in J\right\}$ where each $M_{\alpha}$ is a linear subspace of $Z$. Let ${ }^{*} z_{0}$ be a point in ${ }^{*} Z$ which is not in the closure of ${ }^{*} M$. Then there exists an admissible linear functional ${ }^{*} f$ defined on ${ }^{*} Z$ such that ${ }^{*} f\left({ }^{*} z_{0}\right)=1$ and ${ }^{*} f(u)=0$ for all $u$ in the closure of $* M$.

Proof. It can readily be established that if $z$ is selected from the coset $*_{z}$, then $\left(z(\alpha)+U_{\alpha}\right) \cap M_{\alpha}=\varnothing$ for all $\alpha$ in some member of $\mathscr{U}$. An application of the standard Hahn-Banach theorem yields the desired $* f$.

3.10. The nonstandard strictly singular operators. The above discussion, definitions and theorems are applicable to the situation presented in $\$ 2$. Consider $Z$ to be $\mathscr{L}[X, Y]$ and let $A$ be the subspace of all continuous linear operators of finitedimensional range. It is appropriate to designate $* A$ as the nonstandard continuous linear operators of finite-dimensional range. Theorem 3.7(iii) shows us that the strictly singular operators from $\mathscr{L}[X, Y]$ are characterized as all the standard points in $* \bar{A}$, the closure of the nonstandard operators of finite-dimensional range. We state this result as a theorem.

3.11. THEOREM. Let $H$ denote the class of strictly singular operators in $\mathscr{L}[X, Y]$. Then $H=\mathscr{L}[X, Y] \cap * \bar{A}$.

The standard points of $* A$ are the usual continuous operators of finite-dimensional range. The nonstandard geometric Hahn-Banach theorem (Theorem 3.9) is applicable. A similar situation exists for the strictly cosingular operators [Remark 2.8 ] and the classes of operators mentioned in 2.9 .

We have injected $\mathscr{L}[X, Y]$ into a richer space, its nonstandard model. The situation in the model gives a parallel to the theory of locally convex spaces. A return to $\mathscr{L}[X, Y]$ is quickly accomplished by considering only the standard points in the nonstandard model. When $H$ denotes the strictly singular operators we have $H=\mathscr{L}[X, Y] \cap * \bar{A}$. In this sense we say that the strictly singular operators 
have been characterized as the closure of the continuous linear operators of finitedimensional range for a nonstandard Hausdorff locally convex topology.

\section{BIBLIOGRAPHY}

1. J. W. Brace, The topology of almost uniform convergence, Pacific J. Math. 9 (1959), 643-652. MR 22 \#179.

2. - Approximating compact and weakly compact operators, Proc. Amer. Math. Soc. 12 (1961), 392-393. MR 24 \#A435.

3. - Convergence on filters and simple equicontinuity, Illinois J. Math. 9 (1965), 286-296. MR 31 \#710.

4. J. W. Brace and P. J. Richetta, Approximation of linear operators, Trans. Amer. Math. Soc. 157 (1971), 1-21.

5. C. H. Cook and H. R. Fischer, Uniform convergence structures, Math. Ann. 173 (1967), 290-306. MR 36 \#845.

6. N. Dunford and J. T. Schwartz, Linear operators. I: General theory, Pure and Appl. Math., vol. 7, Interscience, New York, 1958. MR 22 \#8302.

7. H. R. Fischer, Limesräume, Math. Ann. 137 (1959), 269-303. MR 22 \#225.

8. S. Goldberg, Unbounded linear operators: Theory and applications, McGraw-Hill, New York, 1966. MR 34 \#580.

9. R. H. Herman, Generalization of weakly compact operators, Trans. Amer. Math. Soc. 132 (1968), 377-386. MR 36 \#6976.

10. J. Horváth, Topological vector spaces and distributions. Vol. 1, Addison-Wesley, Reading, Mass., 1966. MR 34 \#4863.

11. T. Kato, Perturbation theory for nullity, deficiency, and other quantities of linear operators, J. Analyse Math. 6 (1958), 261-322. MR 21 \#6541.

12. J. L. Kelley, General topology, Van Nostrand, Princeton, N. J., 1955. MR 16, 1136.

13. A. Pełczyński, On strictly singular and strictly cosingular operators. I, II, Bull. Acad. Polon. Sci. Sér. Sci. Math. Astronom. Phys. 13 (1965), 31-41. MR 31 \#1564.

14. A. Robinson, Non-standard analysis, North-Holland, Amsterdam, 1966. MR 34 \#5680.

15. — Nonstandard arithmetic, Bull. Amer. Math. Soc. 73 (1967), 818-843. MR 36 \#1319.

16. H. H. Schaefer, Topological vector spaces, Macmillan, New York, 1966. MR 33 \#1689.

17. F. Treves, Topological vector spaces, distributions and kernels, Academic Press, New York, 1967. MR 37 \#726.

18. Ju. N. Vladimirskiǐ, Strictly cosingular operators, Dokl. Akad. Nauk SSSR 174 (1967), 1251-1252=Soviet Math. Dokl. 8 (1967), 739-740. MR 35 \#7142.

Department of Mathematics, University of Maryland, College Park, Maryland 20742

Systems Evaluation Division, Institute for Defense Analyses, Arlington, Virginia 22202 